may be as small as one micron in diameter. Such particles are products of breakdown or are introduced into the enclosed system at the time of assembly. If special precautions were not taken to eliminate stray particles, the subnormal breakdown strengths obtained would lay within \pm 10 percent of a mean value which for a given method of electrode polishing would be repeatable. These mean values, reported earlier, ${ }^{1}$ are in agreement with the results published by other workers.

Experiments are continuing to determine whether the maximum values reported here are indeed the intrinsic electric strengths of the liquids concerned.

The author is indebted to the National Research Council of Canada for the award of a Fellowship which enabled him to carry out this work.

1W. D. Edwards, Can. J. Phys. 29, 310 (1951).

\section{Rapid Separations of Protactinium and Uranium Radioisotopes from Cyclotron Bombarded} Thorium Nitrate

W. WAyne MeINkE

Department of Chemistry, University of Michigan, Ann Arbor, Michigan (Received February 12, 1952)

$\mathrm{E}^{\mathrm{x}}$ XTENSION of work on collateral chains of the natural radioactive families ${ }^{1-3}$ to short-lived members ${ }^{4}$ has necessitated the development of new rapid chemical procedures for the separation of protactinium and uranium isotopes. Existing target procedures ${ }^{5}$ were adaptable to separation times of 5 to 10 minutes, but new techniques were required for separation times of the order of one minute. Two techniques made these rapid separations possible. First the thorium was bombarded as the thorium nitrate salt in the jiffy-probe of the Berkeley .184-inch cyclotron with a pneumatic tube set-up which delivered the target to the processing area within 12-15 seconds after the end of bombardment. ${ }^{3}$ Secondly, wet coarse filter paper was used as a separating medium for aqueous and organic layers in the extraction process.

To separate protactinium the thorium nitrate target material was emptied into a large funnel containing equal volumes of $4 N$ nitric acid and $0.25 M$ thenoyl trifluoroacetone $(T T A)^{6,7}$ in benzene. The funnel had been fitted with a ground glass stopcock and was lined with wet coarse filter paper. The mixture was stirred vigorously to effect the solution of the target material and to chelate simultaneously the protactinium in the organic layer. After 5 to 10 seconds of stirring (depending upon the protactinium yield desired) the stopcock was opened; the acid layer passed through the filter paper while the benzene layer remained in the funnel. $4 \mathrm{~N}$ nitric acid washes of the benzene layer were used to further remove alpha-emitting radioactive impurities.

Portions of the benzene fraction were then discharged onto platinum counting plates or dishes which were being heated with a heat lamp and hot plate. The samples were then immediately ignited to further speed evaporation. Flaming of the final plate in a Fisher burner flame left a weightless sample of protactinium ready for alpha-counting or alpha-pulse analysis.

Approximate times required for the above steps were solution, extraction, and separation 10-20 seconds; washing 5-10 seconds; and making of sample plate $(\sim 1 \mathrm{cc}$ benzene) $\sim 30$ seconds.

The uranium procedure used the same techniques as above. The thorium nitrate target was emptied into the funnel containing $20 \mathrm{ml}$ of $3 \mathrm{M}$ magnesium nitrate $(0.1 \mathrm{~N}$ in nitric acid) and $8 \mathrm{ml}$ of diethyl ether. Solution and extraction were accomplished in one stirring. Wash solutions of magnesium nitrate removed other alpha-emitting impurities and the last traces of the thorium nitrate target material from the ether fraction. One final wash with ammonium nitrate solution insured that no nonvolatile material would remain in the ether fraction to give "thick" plates for counting. Two pre-extractions of the salting solutions with ether were required to further eliminate non volatile foreign material which would extract into the ether. Ignition of the ether sample gave a weightless plate of the uranium isotopes.

Approximate times required for the steps were: solution, extraction, and separation 10-20 seconds; washing 10-15 seconds; and making of sample plate $(\sim 1 \mathrm{cc}$ ether $) \sim 15$ seconds.

In nuclear chemical work where the main interest is in characterizing particular isotopes, emphasis is on radiochemical purity of the isotope rather than on chemical yield. The above procedures give only a $10-20$ percent chemical yield $-\frac{1}{10}$ of which can be rapidly placed on a plate for counting. These yields are quite satisfactory for the purpose, however, since contaminating isotopes have been reduced by a factor of $10^{4}$ to $10^{5}$. Experimental results have shown that about 1 part in $10^{4}$ of actinium, 1 part in $10^{6}$ of protactinium, and 1 part in $10^{5}$ of thorium come through the uranium procedure. Even this maximum decontamination may prove inadequate, however, when alphabbombardment conditions (energy, beam strength, and contamination by deuterons) are such that the contaminating isotopes are present initially in amounts 100 or more times greater than the uranium isotopes. Similarly the protectinium procedure removes all but about 0.1 percent of the thorium but still has proved too crude for certain branching ratio characterizations of protactinium isotopes.

The method of target solution and separation of organic and aqueous layers by wet filter paper has general applicability for rapid separations of other radioisotopes from their target materials.

The author wishes to thank Mr. C. Kalfian for his help in developing some of the techniques described here and also Professor Glenn T. Seaborg for his encouragement in this work.

1 Ghiorso, Meinke, and Seaborg, Phys. Rev. 74, 695 (1948).

${ }_{2}$ Meinke, Ghiorso, and Seaborg, Phys. Rev. 75, 314 (1949).

3 Meinke, Ghiorso, and Seaborg. Phys. Rev. 81, 782 (1951).

Meinke, Ghiorso, and Seaborg. Phys. Rev. (to be published)

5 W. W. Meinke, AEC Declassified Documents AECD-2738 and AECD 2750 (August, 1949). (August 13, 1947); also J. Am. Chem. Soc. 72, 2948 (1950).

7 ITA is obtainable from Dow Chemical Company, 310 Sansome Street, San Francisco. California.

\section{Determination of the Heat of Dimerization of Tetrafluoroethylene by a Kinetic Method}

Bernard Atkinson AND ANTONy B. TRENwith

Department of Inorganic and Physical Chemistry, Imperial College of Science and Technology, London, S.W, 7, England

(Received February 18, 1952)

D

URING a study of the pyrolysis of tetrafluoroethylene we observed that up to $600^{\circ}$ the rates of the reaction,

$$
2 \mathrm{C}_{2} \mathrm{~F}_{4}=\mathrm{C}_{4} \mathrm{~F}_{8} \text { (octafluorocyclobutane), }
$$

and the reverse reaction,

$$
\mathrm{C}_{4} \mathrm{~F}_{8}=2 \mathrm{C}_{2} \mathrm{~F}_{4} \text {, }
$$

are much faster than the rates of any other homogeneous reactions occurring in the system at normal pressures. In a Pyrex vessel heterogeneous reactions including

$$
\mathrm{C}_{2} \mathrm{~F}_{4}+\mathrm{SiO}_{2}=\mathrm{CO}+\mathrm{SiF}_{4}
$$

take place at a noticeable rate at $550^{\circ} \mathrm{C}$. By using a steel vessel completely freed from iron oxide we have been able to show that an equilibrium

$$
2 \mathrm{C}_{2} \mathrm{~F}_{4} \rightarrow \mathrm{C}_{4} \mathrm{~F}_{8}
$$

is readily established at $550^{\circ} \mathrm{C}$. The pressure change on heating perfuorocyclobutane at $520-590^{\circ} \mathrm{C}$ is in accord with that for a first-order reaction moving to equilibrium with a second-order reaction.

We have measured the rate of reaction (1) in a Pyrex vessel at temperatures from $300-550^{\circ} \mathrm{C}$ and the rate of reaction (2) in a $\mathrm{Py}$ rex vessel at $520-550^{\circ} \mathrm{C}$ and in a steel vessel at $550-590^{\circ} \mathrm{C}$. Values 
of the rate constant for reaction (2) at $550^{\circ} \mathrm{C}$ calculated from the results obtained with the Pyrex vessel agreed with those obtained using the steel vessel. All measurements were made at $100-500 \mathrm{~mm}$ pressure.

Plots of $\log k$ against $I / T$ for both the forward and back reactions gave straight line graphs. Equilibrium constants were calculated from ratios of rate constants, and the heat of reaction for (1) was derived from a graph of $\log K$ against $I / T$. For the forward reaction (1) the results gave the constants in the equation $k=P Z e^{-E / R T}$ as $E=25.4 \mathrm{kcal}, P=3.85 \times 10^{-4}$. For the reverse reaction, taking $k=A e^{-E / R T}, E=74.1 \mathrm{kcal}, A=8.9 \times 10^{15} \mathrm{sec}^{-1}$. The figures for the equilibrium constant gave $\Delta H=-49.8 \mathrm{kcal}$, $\Delta S=-47.4 \mathrm{cal}$ per deg, both figures being for $550^{\circ} \mathrm{C}$ approximately.

The energy of activation for the forward reaction is distinctly lower than that for the polymerization of ethylene to butylene and higher olefines. For the latter reaction Pease ${ }^{1}$ found $E=35$ kcal, $P=5 \times 10^{-4}$. Both our figure for the steric factor of reaction (1) and the figure of Pease for ethylene give support to the theory that association reactions between polyatomic molecules will in general have small steric factors. ${ }^{2}$ The $A$ factor found for the reverse reaction is similar to that for other reactions involving ring opening, thus for cyclopropane $A=1.48 \times 10^{15} \mathrm{~cm}^{-13}$ and for trioxymethylene $A=1.43 \times 10^{16} \mathrm{~cm}^{-1}$. The $^{4}$ factor is much above the "normal" value of $10^{13}-10^{14} \mathrm{~cm}^{-1}$.

From thermochemical data we estimate that for the reaction

$$
2 \mathrm{C}_{2} \mathrm{H}_{4}=\mathrm{C}_{4} \mathrm{H}_{8} \text { (cyclobutane), }
$$

the value of $\Delta H$ is $-25 \mathrm{kcal}$. As the cyclobutanes contain twelve bonds and the reacting olefine molecules ten bonds, the difference between this figure and that given above for reaction (1) cannot be said to indicate any great structural differences between the fluorocarbons and hydrocarbons involved.

A full account of experimental methods and results is being prepared.

We thank I.C.I. Ltd. for the gift of tetrafluoroethylene, the University of London for a grant towards the cost of apparatus, and the D.S.I.R. for a maintenance grant to one of us (A.B.T.).

1 R. N. Pease, J. Am. Chem. Soc. 53, 613 (1931).

2 C. E. H. Bawn, Trans. Faraday Soc. 31,1536 (1935)

T. S. Chambers and G. B. Kistiakowsky, J. Am. Chem. Soc. 56, 399 (1934).

1 R. LeG. Burnett and R. P. Bell, Trans. Faraday Soc, 34, 420 (1938).

\section{The Microwave Absorption Spectrum of Acetone Vapor*}

Thomas L. WeatherLy aNd DUdLEy Williams Department of Physics and Astronomy, The Ohio Stale University, Columbus, Ohio

(Received February 6,1952 )

$\mathrm{T}$ HE microwave spectrum of acetone vapor in the spectral region between 18,000 and $30,000 \mathrm{mc} / \mathrm{sec}$ has been examined. The observed spectrum includes the four strong lines whose frequencies are listed in Table $\mathbf{I}$. The seventeen lines in

TABLE I. Strong lines in the microwave spectrum of acetone.

$26,661.5 \mathrm{mc} / \mathrm{sec}$
$28,340.0$
$28,622.3$
30,070

the region 22,000 to $25,000 \mathrm{mc} / \mathrm{sec}$ reported by Bak et al. ${ }^{1}$ were not observed in the present study and hence must be much weaker than the lines listed in the table.

An attempt to interpret the observed lines as transitions between the rotational energy levels of an asymmetric top has been unsuccessful. The asymmetry parameter $K=0.4$ for the acetone molecule if the interatomic distances and bond angles have values similar to those in related molecules. ${ }^{2}$ The spectrum predicted for the region between 18,000 and $30,000 \mathrm{mc} / \mathrm{sec}$ on the basis of
$K=0.4$ includes 23 lines for rotational transitions involving $J$ values no higher than $J=6$. Moreover, the energy level populations and transition probabilities are such that one can expect relatively intense lines in this region for $J$ values as high as 30 . Thus, there seems little hope of positively identifying the strong lines observed. Although the lines were observed with a spectrograph employing ground-biased square wave Stark effect modulation, no clearly defined Stark patterns were observed; this probably indicates that the rotational transitions are between levels with high $J$ values, since in this case the number of Stark components would be larger and the intensities of the individual components would be small. However, the behavior of the observed lines, when the wave guide was cooled with dry ice, indicates that the $J$ values involved are less than $J=35$.

The intensities of the lines found in the present study are so much greater than those of the lines reported by Bak $e$ al al. ${ }^{1}$ that they will probably prove to be the lines most suited for microwave spectrochemical analysis.

* This work was supported in part by a contract between the Geophysical Division of the Air Force Cambridge Research Center and the Ohio State University Research Foundation.

1 Bak, Knudsen, and Madsen, Phys. Rev. 75, 1622 (1949).

2 G. Herzberg, Infrared and Raman Spectra (D. Van Nostrand Company. Inc., New York, 1945), p. 440 .

\section{X-Ray Diffraction Study of Crystalline Neopentane (Tetramethyl Methane)*}

Arthur H. Mones and Benjamin Post Polytechnic Institule of Brooklyn, Brooklyn, New York (Received February 18, 1952)

$\mathrm{N}$ the basis of an optical study at $-180^{\circ} \mathrm{C}$, Wahl ${ }^{1}$ reported that crystalline neopentane is birefringent at that temperature and probably tetragonal. He also noted that the crystals underwent a transition to an isotropic form well below the melting point $\left(\mathrm{mp}=-19.7^{\circ} \mathrm{C}\right)$. The existence of this transition has since been confirmed by Aston et al. ${ }^{2}$ Some time after Wahl's observations were published, Mark and Noethling ${ }^{3}$ reported (on the basis of Debye-Scherrer diagrams taken at the temperature of liquid air) that neopentane crystallized in the cubic system with $a_{0}=11.26 \mathrm{~A}$ and with 8 molecules per unit cell.

In this communication some results of an $x$-ray diffraction study of crystalline neopentane, above and below the transition temperature, are reported. The low temperature $\mathrm{x}$-ray camera used in this investigation has been described elsewhere. ${ }^{4}$

Powder diagrams of the crystalline phase, stable above the transition temperature $\left(-143^{\circ} \mathrm{C}\right)$, were not satisfactory. Only three or four very broad lines were observed. Single crystals of neopentane were then grown in thin-walled glass capillary tubes and oscillation diagrams were obtained at $-50 \pm 5^{\circ} \mathrm{C}$. In this temperature region the crystals appeared isotropic. The $x$-ray diagrams were indexed on the basis of a face-centered cubic unit cell with $a_{0}=8.78 \pm 0.05 \mathrm{~A}$ and with four molecules per unit cell.

Intensities of reflections decreased very rapidly with increasing Bragg angles. Reflections were observed only from (111), (200), (220), (311), (222), (400), and (333). The large entropy of transition, 4.39 eu per mole, compared with an entropy of fusion of 3.03 eu per mole, indicates considerable disorder in the crystal above the transition temperature. The $\mathrm{x}$-ray evidence, however, is too meagre to permit any definite conclusions to be drawn concerning the nature of the disorder. It may be noted, however, that structure factors computed on the assumption of random orientation of neopentane molecules above lattice points, with spherical symmetry on the average, showed good agreement with "observed" structure factors. Structure factors calculated, assuming the following models, showed poor agreement with observed structure factors: (a) an ordered structure; and (b) a disordered structure, with the four tetrahedrally disposed carbon atoms of each molecule occupying, statistically, the eight corners of a cube.

Single crystals of the high temperature form invariably broke 